\title{
NK/T-Cell Lymphoma: A Nasal Nightmare
}

\author{
${ }^{1}$ Rahul R Gupta, ${ }^{2}$ RG Aiyer, ${ }^{3}$ Prarthna J Jagtap, ${ }^{4}$ Jaimin Patel
}

\begin{abstract}
Nasal and paranasal lymphomas usually present an intricate diagnostic challenge considering its lack of specific signs and symptoms. In Indian setup patients usually present in late stages of the disease. Brain involvement leads to the treatment being unfruitful as the patient deteriorates were fast. We are presenting our 12-year-old tribal patient's case details in whom the terminal stage of presentation led to convulsions, respiratory failure and death. Delay in pathological and immunohistochemistry diagnosis probably also was a factor leading to delay in starting chemotherapy. Diagnostic and treatment dilemmas considering the patients socioeconomic class and general nutrition and health are also considered.
\end{abstract}

Keywords: Lymphomas, NK/T cell, Nose and PNS, Management.

How to cite this article: G upta R R, Aiyer R G, J agtap PJ , Patel J. NK/T-Cell Lymphoma: A Nasal Nightmare. Clin Rhinol An Int J $2014 ; 7(1): 23-25$.

\section{Source of support Nil}

\section{Conflict of interest None}

\section{INTRODUCTION}

Natural killer (NK) cell lymphomas are rare malignancies. They are classified as nasal type extranodal NK/T-cell lymphoma and aggressive NK cell leukemia. ${ }^{1}$

Extranodal nasal-type natural killer NK /T-cell lymphoma is a distinct entity according to the World $\mathrm{H}$ ealth O rganization (WHO) classification of lymphoid tissue. ${ }^{2}$

Extranodal NK/T-cell lymphoma nasal type, is a rare type of non-Hodgkin lymphoma. It is more common in A sian and South-A merican countries. ${ }^{3}$ Extranodal NK/T-cell lymphoma nasal type, can develop in either T cells or natural killer (NK) cells, but most often in the NK cells.

Extranodal NK/T-cell lymphoma, nasal type has a predominant tendency to occur in young males. ${ }^{2}$

M ost cases of $\mathrm{NK} / \mathrm{T}$-cell lymphoma originate from the nasal cavity, hence the term nasal NK/T-cell lymphoma. Tumors resembling the prototype of nasal NK/T-cell lym-

\footnotetext{
${ }^{1}$ Assistant P rofessor, ${ }^{2}$ P rofessor and Head ${ }^{3}$ Associate Professor, ${ }^{4} \mathrm{~J}$ unior Resident

${ }^{1-4}$ Department of ENT, Head and Neck Surgery, Government Medical College and SSG Hospital, Vadodara, Gujarat, India
}

Corresponding Author: RG Aiyer, Professor and Head Department of ENT, Head and Neck Surgery, 3/2 J esal Apartment Abhishek Colony, Racecourse, Vadodara-7, Gujarat, India Phone: 9825184648, e-mail: drrgaiyer@ hotmail.com phoma occurring in a variety of extranasal sites are referred to as nasal-type NK/T-cell lymphoma. ${ }^{2}$

The primary sites of 'nonnasal' nasal-type NK/T-cell lymphoma vary according to different definitions and categorization, but the majority occurs in the upper aerodigestive tract. A lthough well recognized, the characteristics and optimal therapy of nasal-type NK/T-cell lymphoma are largely unknown. ${ }^{2}$

Nasal NK/T-cell lymphomas present mostly with stage I/II disease. Concomitant/sequential chemotherapy and radiotherapy is standard treatment. Radiotherapy alone is inadequate because of high systemic failure rate. For stage III/IV nasal, nonnasal, and disseminated lymphomas, systemic chemotherapy is indicated. Regimens containing I-asparaginase and drugs unaffected by P-glycoprotein are most effective. ${ }^{3}$

\section{CASE REPORT}

A 12 years old tribal boy coming from lower socioeconomic class residing at a far off village in Vadodara district of Central Gujarat presented to us with c/o ulcerative lesion over dorsum of nose and palate with complaints of:

- Disfiguring erosion of nose for 1.5 to 2 months.

- Foul smelling discharge for 1 to 1.5 months.

- Edematous swelling of perinasal region for 1 month.

- Low grade fever since 25 days.

- Weakness of right upper and lower limb for 10 to 12 days.

\section{EXAMINATION FINDINGS (Figs 1 to 4 )}

- Destruction of external nose: cartilaginous septum, both sides upper and lower lateral cartilages of nose.

- Blackening of skin and subcutaneous tissue with slough and necrosis around it. Edematous swelling of nasolabial fold and paranasal soft tissue.

- Maxillary sinus was exposed on both side because of erosion of anteromedial bony and mucoperiosteal structures. Edematous and congested remnant nasal mucosa with few spots of purul ent discharge.

- Ophthalmologists ruled out the presence of any sclerokeratosis and abnormality in eyeball movements.

- Hematological and serological investigations revealed anemia ( $\mathrm{Hb}: 9.1 \mathrm{gm} / \mathrm{dl}$ ), thrombocytosis (6.08 lacs/ cumm), lymphopenia with a total count of $11,700 / \mathrm{cmm}$ and a nonreactive S. HIV and S. HBsA g. Electrolytes 


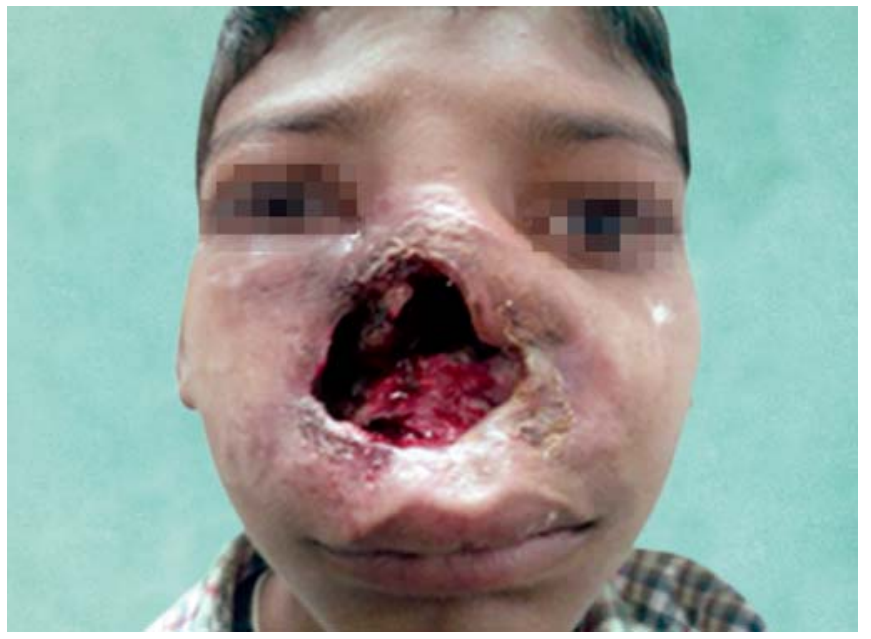

Fig. 1: Complete necrosis of external nose with perilesional edema

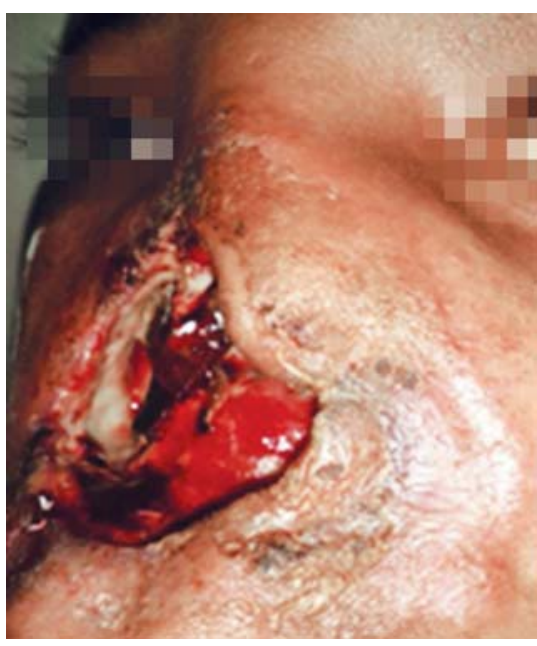

Fig. 3: Left lateral view

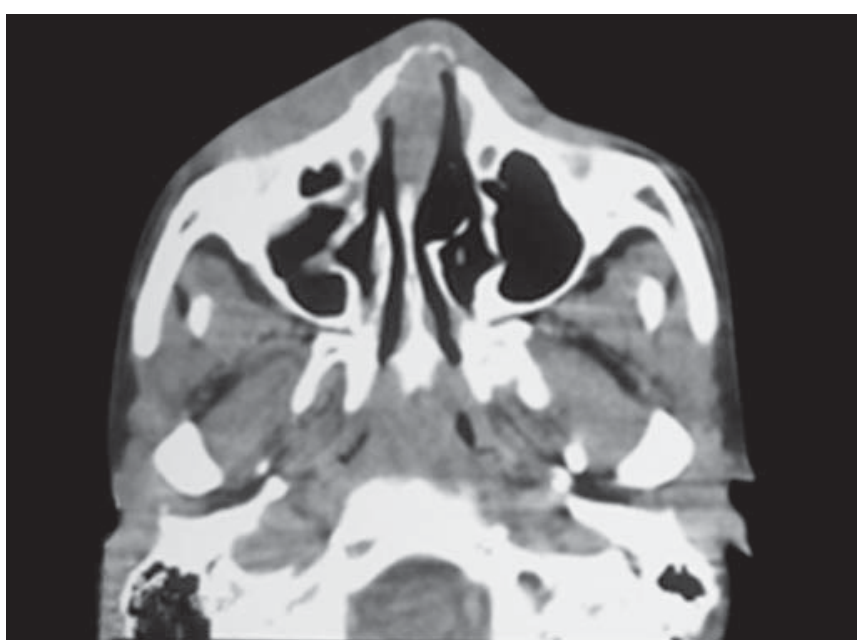

Fig. 5: CT scan showing grossly normal sinuses

were also within normal limits $\left(\mathrm{S} . \mathrm{Na}^{+}: 130 \mathrm{mM}\right.$ ol/l and $\mathrm{S} . \mathrm{K}^{+}: 5.7 \mathrm{mM}$ ol/l). A blood sample was sent for C-ANCA and P-ANCA immunofluorescence was reported negative for this study.

- U rine routine and microscopic examination did not reveal any abnormality other than the presence of occasional.

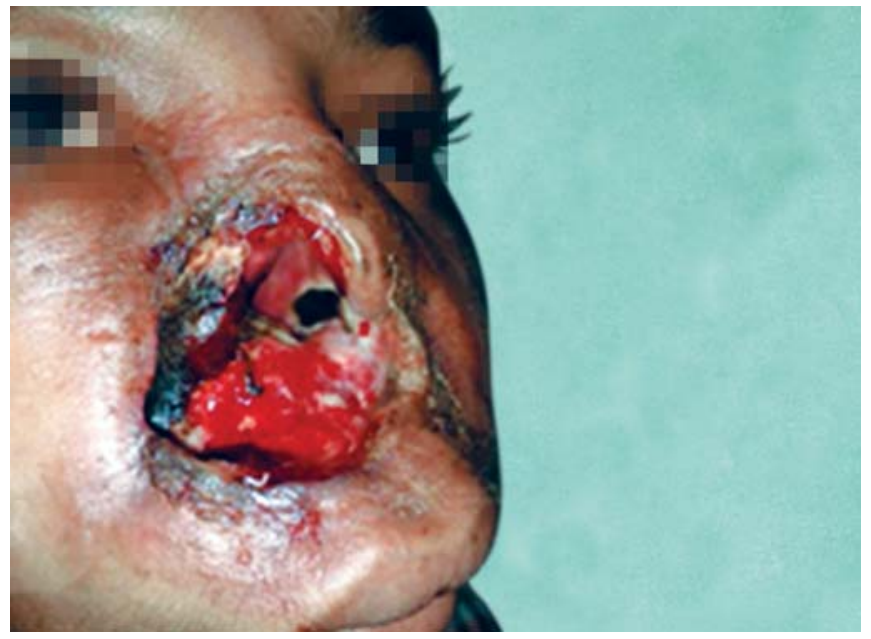

Fig. 2: Erosion of medial of left maxillary sinus

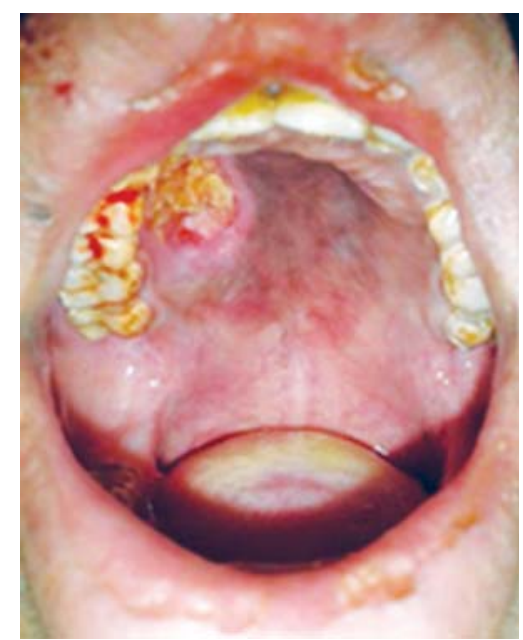

Fig. 4: Palatal necrosis

- No abnormality was detected on chest roentgenogram and abdominal ultrasonography.

- Culture of purulent discharge from PNS did not grow any bacteria or fungi.

- A Computerized Tomogram of Paranasal Sinuses with I/V contast revealed an inflammatory/infective lesion in b/l premaxillary regi on involving both nasal fossae and al ae of nose. Cranially, thelesion was extending into extraconal compartment of right orbit (inferomedially) and caudally up to mandible. Even though right osteomeatal complex was blocked, there was very minimal mucosal disease in right maxillary, sphenoid and both anterior ethmoid sinuses. Left middle turbinate had a concha bullosa and mild deviation of nasal septum to right (Fig. 5). M RI brain revealed multifocal poorly defined, irregular shaped, abnormal signal intensity lesions in corticalsubcortical location of bilateral frontal, bilateral parietal, bilateral temporal, left occipital and left cerebellum with irregular gyromeningeal enhancement within mild to moderate perilesional edema (Fig. 6). Going by the clinical profile of the patient radiologists quoted possibilities of 

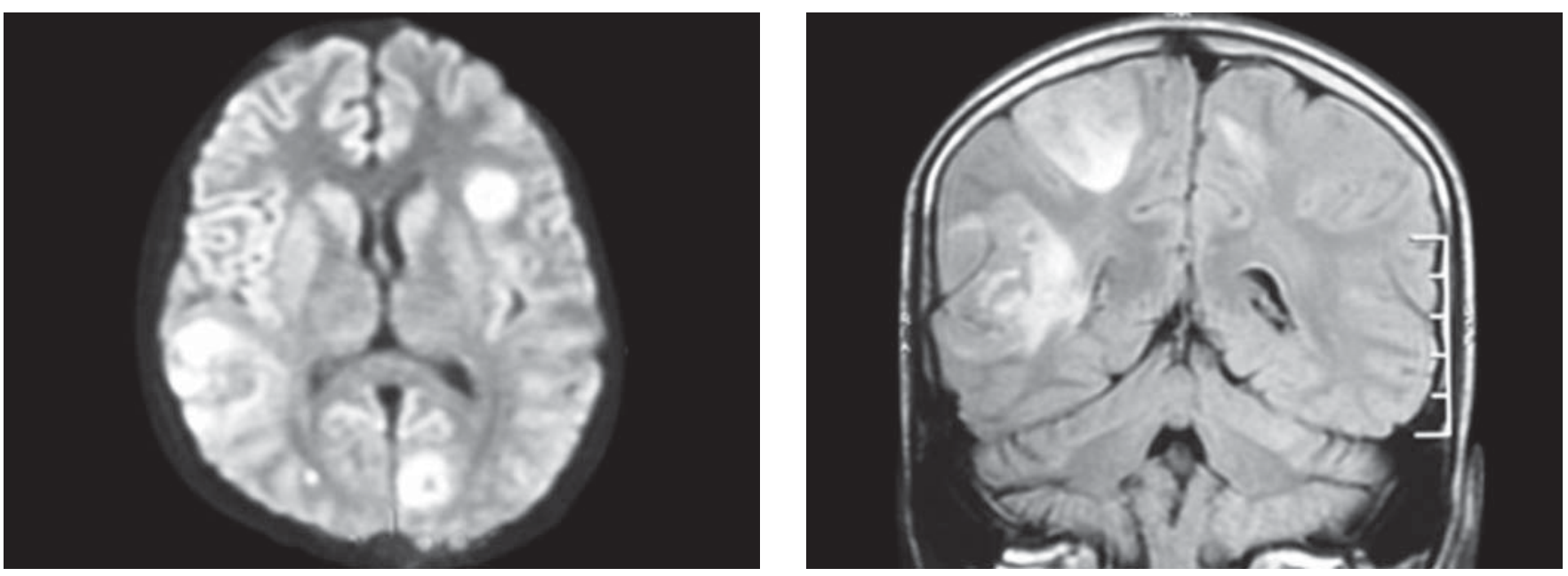

Fig. 6: Multiple intracranial lesions

typical granulomatous lesions, lymphomatous lesions or fungal granulomas.

A biopsy taken from granulation tissue present at mucosal and cutaneous margins of lesion narrowed down our diagnostic dilemma to NK/T cell lymphoma or Wegener's granulomatosis. Slides showed well defined aggregates of histiocytes, angiocentric aggregates of cells, occasional giant cells, necrosis and dense suppurative inflammation.

Immunohistochemistry done on the biopsy specimen was strongly positive for P53, cytoplasmic CD3 and negative for CD56.

All these features confirmed the diagnosis of nasal type NK/T cell lymphoma.

$M$ edical oncologists advised a chemotherapy regimen of six cycles of CHOP (Cyclophosphamide $800 \mathrm{mg}$, Vincristine $60 \mathrm{mg}$ and A driamycin $60 \mathrm{mg}$ ) regimen at the interval of 21 days.

Patient was given first cycle of this regimen with adequate post chemotherapy hydration. Post chemo routine hematogram, renal and liver functions were normal.

Two days after chemotherapy, patient suddenly developed aphasia and became disoriented; patient developed generalized tonic clonic convulsion for which he was immediately shifted to M ICU and was put on anticonvulsant therapy. A fter 12 hours patient became unconscious, lost spontaneous respiration for which he was intubated and put on ventilator. Cerebrospinal fluid microscopic examination and culture did not reveal any abnormality. A fter a couple of days a tracheostomy was also performed uneventfully. We lost the patient after a day as all resuscitation attempts failed.

\section{DISCUSSION}

$M$ alignant lymphomas of the head and neck region that originate in the nasal cavity, paranasal sinuses and hard palate form an interesting and frequently diagnostically difficult group. In the past, these lymphomas have been confused with a number of infectious, autoimmune, or inflammatory designations, most of which we now know represent peripheral T-cell lymphomas or angiocentric immunoproliferative lesions. These older terms include midline lethal granuloma, polymorphic reticulosis and midline malignant reticulosis. Even after correct diagnosis long-term survival is still restricted due to the late stages of presentation of our patients. Poor nutritional status also increases the number of post chemotherapy complications likely in our set of patients.

N onspecific nasal symptoms often predate the appearance of mucosal ulceration and tissue necrosis by 1 year or more. The ambiguous nature of these symptoms can result in a delay in diagnosis unless a high index of suspicion is maintained uptill final diagnosis by IHC. Representative biopsy material and excellent communication with the pathologist is also important. A lthough not al ways possible, a diagnosis should be sought prior to commencing a treatment course. It needs to be understood that the term midline lethal granul oma should be a clinical term used only until specific laboratory or histological diagnosis is obtained, and not form a basis for therapy. In conclusion, clinicians should consider extranodal nasal lymphoma as a rare cause of midline destructive lesions. They should be aware of the difficulties of obtaining histological diagnosis despite apparently adequate biopsies.

\section{REFERENCES}

1. Kwong $Y L$. The diagnosis and management of extranodal NK/T-cell Iymphoma, nasal-type and aggressive NK-cell leukemia. US National Library of M edicineJ Clin Exp Hematop. 2011;51(1):21-28.

2. Ye-Xiong Li, Hui Fang, Qing-Feng Liu. Clinical features and treatment outcome of nasal-type NK $/ \mathrm{T}$-cell lymphoma of Waldeyer ring. Blood 2008 Oct 15;112(8)3057-3064.

3. Tse $E, K$ wong $Y L$. How I treat NK/T-cell Iymphomas. US National Library of M edicine. Blood. 2013 Jun 20;121(25):49975005. Epub 2013 M ay 7. 